\author{
Min-Shin Chen \\ National Taiwan University, \\ Department of Mechanical Engineering, \\ Taipei, Taiwan
}

\title{
A Tracking Controller for Linear Time-Varying Systems
}

This paper proposes a new tracking control design for linear time-varying systems. The proposed control input, which is in the span of finitely many preselected input data, minimizes the $L_{2}$-norm of the output tracking error. The more input data is used, the less $L_{2}$-norm of the tracking error is achieved. The design of the new controller, which consists of a feedforward controller and a discretized state feedback loop, requires a finite-time preview of the system parameters and the reference trajectory. It is shown that as long as the preview time is longer than a critical value, the closed-loop stability is maintained irrespective of the stability property of the system's zero dynamics. When the system parameters are periodically time-varying, the proposed design can be solely based on a set of experimental input and output data instead of on the exact information of system parameters.

\section{Introduction}

The tracking control design for systems with unstable zero dynamics (Isidori, 1989) presents difficult challenge mainly due to the fact that the system model must be inversed in order to track an arbitrary reference trajectory exactly. As a result, conventional tracking control designs such as the model following control require an unbounded input signal to achieve exact tracking when the inversed system is unstable. Since many practical systems have unstable zero dynamics, it is of great importance that new tracking control designs be explored so that exact or approximate tracking can be achieved for systems with unstable zero dynamics using only bounded input.

For the tracking control of linear time invariant systems with unstable zero dynamics, Tomizuka (1987) proposes an approximate tracking controller, which uses feedforward compensation to ensure a zero-phase tracking error. Later researches, (see Haack and Tomizuka, 1991; Menq and Xia, 1990; and Gross et al., 1994) further reduce the gain error of the zero-phase tracking controller using more complex feedforward compensations. For linear time-varying systems, most tracking control designs such as those proposed by Arvanitis and Paraskevopoulos (1992) and Tsakalis and Ioannou (1993) require the zero dynamics of the system to be stable. One exception that does not require the stable zero dynamics assumption appears in Kamen (1989) where the reference trajectory is generated from some known stable exosystem. Under this assumption, the internal model principle is employed to achieve asymptotic tracking. For arbitrary reference trajectories, Zhao and Chen (1994) utilize the preview concept and propose a stable inversion algorithm for nonlinear time invariant systems with unstable zero dynamics. Benedetto and Lucibello, (1993), and Devasia and Paden (1994) introduce preview tracking control designs for time-varying nonlinear systems with unstable zero dynamics. However, the tracking operation time is confined to be finite. No algorithm is available yet for the infinite-time tracking control of time-varying systems with unstable zero dynamics.

This paper proposes a new tracking control for linear timevarying systems with unstable zero dynamics. The new control input is in the span of a finite number of preselected input data, and the optimal linear combination of such input data is determined by the parameter optimization principle. Similar

Contributed by the Dynamic Systems and Control Division for publication in the Journal, of Dynamic Systems, Measurement, and Control. Manuscrip received by the DSCD November 28, 1994; revised manuscript received August 26, 1995. Associate Technical Editor: Tsu-Chin Tsao. approaches can be found in the finite-time state transferring control in Choura (1992) and the suboptimal $L Q$ regulation control for linear time-varying systems by Kleinman and Athans (1968). The design proposed in this paper has the following advantages compared with previous designs: (1) The proposed design can be applied to the infinite-time tracking control with the closed-loop stability ensured. (2) The size of the tracking error can be controlled by the number of input data used. The more input data is used, the smaller the tracking error is achieved. (3) When the system parameters vary periodically, the proposed design does not require information of time-varying system parameters. Instead, it requires only a set of experimental input and output data to generate the optimal control signal. In other words, there is no need for parameter identification in the proposed design when the system is periodical.

\section{Problem Formulation}

Consider a multivariable Linear Țime-Varying (LTV) system

$$
\begin{aligned}
\dot{x}(t)=A(t) x(t) & +B(t) u(t), \quad x(0)=x_{0} \\
y(t) & =C(t) x(t),
\end{aligned}
$$

where $x(t) \in R^{n}$ is the system state, $u(t) \in R^{m}$ the control input, $y(t) \in R^{n}$ the system output, and $A(t), B(t)$, and $C(t)$ system matrices of appropriate dimensions. In this paper, only the tracking control design will be treated, and it is assumed that the regulation control design has been done in the sense that there exist $m$ and $\sigma \in R^{+}$such that

$$
\|\Phi(t, \tau)\| \leq m e^{-\sigma(t-\tau)}, \quad \forall t>\tau,
$$

where $\Phi(t, \tau)$ is the state transition matrix of the free system (1). The control problem is as follows. Given a bounded reference trajectory $y_{m}(t)$, find a control input of the following form

$$
u(t)=\sum_{i=1}^{N} \theta_{i} u_{i}(t),
$$

which can drive the system output $y(t)$ to track a reference trajectory $y_{m}(t)$ as closely as possible, where $u_{i}(t)$ 's are preselected input data, and $\theta_{i}^{\prime} s$ are constant parameters to be determined. Two different cases of tracking problems are discussed in this paper: (1) finite-time tracking control, where the reference trajectory $y_{m}(t)$ is defined over a finite time interval [0, $T]$, (2) infinite-time tracking control, where $y_{m}(t)$ is defined over the infinite time interval $[0, \infty)$.

Throughout this paper, given two matrices $f(t)$ and $g(t)$ with compatible dimensions, denote $\langle f, g\rangle$ by 


$$
\langle f, g\rangle \triangleq \int_{0}^{T} \omega(\tau) f^{T}(\tau) g(\tau) d \tau,
$$

where $\omega(\cdot)$ is a non-negative weighting function, and $T$ a time interval length. It is noted that $\langle f, g\rangle$ may be a scalar, a vector, or a matrix, depending on the dimensions of $f(t)$ and $g(t)$.

\section{Finite-Time Tracking Problem}

For the finite-time tracking control problem, it is assumed that the information of $A(\tau), B(\tau)$, and $C(\tau), \tau \in[0, T]$ is known a priori at time $t=0$. With this information, the following output response data can be obtained by performing numerical integration with Eq. (1): (I) A set of zero-input responses $£_{0}\left(x_{0 i}\right)(t)^{\prime} s, t \in[0, T], i=1,2, \ldots, n$ (= system dimension) corresponding to the initial condition $x(0)=x_{0 i}$, where the $n$ initial states $x_{0 i}$ 's are chosen to be linearly independent vectors in $R^{n}$. From linearity of the system (1), given an arbitrary initial state $x_{0}$, its corresponding zero-input response is found to be

$$
\mathrm{Ł}_{0}\left(x_{0}\right)=c_{1} \mathrm{七}_{0}\left(x_{01}\right)+c_{2} \mathrm{七}_{0}\left(x_{02}\right)+\ldots+c_{n} \mathrm{七}_{0}\left(x_{0 n}\right),
$$

where $c_{i}$ 's are determined from the equation $x_{0}=c_{1} x_{01}+c_{2} x_{02}$ $+\ldots+c_{n} x_{0 n}$. (II) A set of zero-initial-state responses $y_{i}^{0}(t)^{\prime} s=L_{u}\left(u_{i}\right)(t)^{\prime} s, t \in[0, T], i=1,2, \ldots, N$ corresponding to the preselected input data function $u_{i}(t)$ in Eq. (3). The input data functions $u_{i}(t)^{\prime} s$ are chosen so that $\left\{y_{i}^{0}(t), i=1\right.$, $2, \ldots, N\}$ are linearly independent time functions over the time interval $[0, T]$. In the sequel, $y_{i}^{0}(t)$ is called the output data and $u_{i}(t)$ the input data.

The proposed tracking control design is based on the minimization of the following cost function

$$
J(\theta)=\langle e, e\rangle=\int_{0}^{T} \omega(\tau)\|e(\tau)\|^{2} d \tau,
$$

where $\omega(\cdot)$ is a weighting function, and $e(t)$ the tracking error

$$
e(t)=y_{m}(t)-y(t) .
$$

The cost function is the weighted $L_{2}$ norm of the tracking error over the time interval $[0, T]$. The goal is to find the optimal parameters $\theta_{i}^{\prime} s, i=1,2, \ldots, N$, in Eq. (3) so that the cost function $J(\theta)$ is minimized.

In what follows, an explicit expression for $J(\theta)$ will be derived. Given an initial state $x_{0}$, the system's input-output relationship is obtained from Eq. (1)

$$
\begin{aligned}
y(t) & =C(t) \phi(t, 0) x_{0}+C(t) \int_{0}^{t} \phi(t, \tau) B(\tau) u(\tau) d \tau \\
& \triangleq \mathrm{七}_{0}\left(x_{0}\right)+\mathrm{七}_{u}(u),
\end{aligned}
$$

where $\mathrm{E}_{0}(\cdot)$ is the zero-input response, and $\mathrm{E}_{u}(\cdot)$ is the zeroinitial-state response. Express the control (3) in the vector form

$$
u(t)=U(t) \Theta,
$$

where $\Theta=\left[\theta_{1}, \theta_{2}, \ldots, \theta_{N}\right]^{T} \in R^{N}$ and $U(t)=\left[u_{1}(t), u_{2}(t)\right.$, $\left.\ldots, u_{N}(t)\right] \in R^{m \times N}$. Substituting the control into Eq. (7) yields $y(t)=\mathrm{Ł}_{0}\left(x_{0}\right)+\mathrm{Ł}_{u}(U \Theta)=\mathrm{Ł}_{0}\left(x_{0}\right)+\mathrm{Ł}_{u}(U) \Theta=\mathrm{Ł}_{0}\left(x_{0}\right)+$ $Y_{0} \Theta$, where $Y_{0} \triangleq \mathfrak{Ł}_{u}(U)=\left[y_{1}^{0}(t), y_{2}^{0}(t), \ldots, y_{N}^{0}(t)\right] \in R^{m \times N}$ with $y_{i}^{0}(t)=L_{u}\left(u_{i}\right)(t)$. The tracking error $(6)$ then becomes

$$
e(t)=y_{m}(t)-\mathbf{L}_{0}\left(x_{0}\right)-Y_{0} \Theta=y_{m 0}(t)-Y_{0} \Theta,
$$

where

$$
y_{m o}(t)=y_{m}(t)-\mathbf{L}_{0}\left(x_{0}\right),
$$

in which $L_{0}\left(x_{0}\right)$ can be calculated from Eq. (4) based on the zero-input response data $L_{0}\left(x_{0 i}\right)^{\prime} s$. The cost function (5) can now be expressed as

$$
\begin{aligned}
J(\Theta) & =\left\langle y_{m 0}-Y_{0} \Theta, y_{m 0}-Y_{0} \Theta\right\rangle \\
& =Y_{m 0}-2 V_{0} \Theta+\Theta^{T} P_{0} \Theta,
\end{aligned}
$$

where $Y_{m 0}=\left\langle y_{m 0}, y_{m 0}\right\rangle \in R$ is a scalar, $V_{0}=\left\langle y_{m 0}, Y_{0}\right\rangle \in R^{1 \times N}$ a row vector, and $P_{0}=\left\langle Y_{0}, Y_{0}\right\rangle \in R^{N \times N}$ a square matrix. The optimal parameter $\Theta$ which minimizes Eq. (9) is given by the following theorem.

Theorem 1: Consider the system (1) subject to the constraint (3). If the output data $\left\{y_{i}^{0}(t), i=1,2, \ldots, N\right\}$ are linearly independent on $[0, T]$, the optimal control which minimizes the cost function (4) is given by

$$
u^{*}(t)=U(t) \Theta^{*}, \quad \Theta^{*}=P_{0}^{-1} V_{0}^{T},
$$

and the minimal tracking error achieved by $u^{*}(t)$ is

$$
e^{*}(t)=y_{m 0}-Y_{0} \Theta^{*}
$$

Proof: See Appendix A.

Although Theorem 1 gives the minimal tracking error (11) with the proposed control (3), it is not clear how good the tracking performance is. In the next theorem, it will be shown that the quality of tracking performance is actually controlled by how many terms of input data functions $u_{i}(t)$ 's are used in Eq. (3).

Theorem 2: The optimal cost $J_{N}^{*}$ is a monotonically decreasing function of $N$; i.e.,

$$
J_{N+1}^{*}<J_{N}^{*} \text { for all } N \text {, if }\left\langle y_{N+1}, e_{N}^{*}\right\rangle \neq 0,
$$

where the subscript $N$ is the number of input data functions used in the control (3). Furthermore,

$$
J_{N}^{*} \rightarrow 0 \text { as } N \rightarrow \infty,
$$

if the output data functions $y_{i}(t), i=1,2, \ldots, \infty$ are complete in the $L^{2}$ space on $[0, T]$.

Proof: See Appendix B.

According to Eq. (12), the more input data functions $u_{i}$ 's are used in the control (3), the less $L_{2}$-norm of the tracking error is achieved. However, Hypothesis in Eq. (12) says that if the new output data $y_{N+1}(t)$ is orthogonal to the previous minimal tracking error $e_{N}^{*}(t)$ in Eq. (11), adding a new input data $u_{N+1}(t)$ in the control input (3) will not make any contribution in decreasing the $L_{2}$-norm of the tracking error. Equation (13) then states that as the number of input data functions approaches infinity, arbitrarily good tracking performance can be achieved in the sense of Eq. (13). Notice that this result is obtained regardless of the stability property of the system's zero dynamics.

\section{Infinite-Time Tracking Problem}

In this section, the finite-time tracking control in Theorem 1 will be applied to the infinite-time tracking control problem. First, the time axis is sliced into infinitely many time intervals, each spanning $T$ seconds, and during each time interval, the finite-time tracking control in Theorem 1 is applied. The proposed infinite-time tracking control is therefore of the following form

$$
u(t)=\sum_{i=1}^{N} \theta_{i}(k) u_{i}(t)=U(t) \Theta(k), \quad k T \leq t<(k+1) T, \begin{aligned}
k & =0,1,2, \ldots
\end{aligned}
$$

where each parameter $\Theta(k)$ minimizes the $L_{2}$-norm of the tracking error on its corresponding time interval 


$$
J_{k}(\Theta(k))=\int_{k T}^{(k+1) T} \omega(\tau)\|e(\tau)\|^{2} d \tau
$$

The same procedure as in Section 3 can now be used to find the optimal parameter $\Theta(k)$ minimizing $J_{k}$, giving

$$
\Theta *(k)=P_{k}^{-1} V_{k}^{T} \text {. }
$$

In Eq. (15), $V_{k}=\left\langle y_{m k}, Y_{k}\right\rangle$ and $P_{k}=\left\langle Y_{k}, Y_{k}\right\rangle$, where $Y_{k}=$ $\left[y_{1}^{k}(t), y_{2}^{k}(t), \ldots, y_{N}^{k}(t)\right]$ with $y_{i}^{k}(t)=L_{u}\left(u_{i}\right)(t), t \in[k T$, $k T+T]$, being the zero-initial-state response subject to the input data $u_{i}(t)$, and

$$
y_{m k}(t)=y_{m}(t)-€_{0}(x(k T)),
$$

with $L_{0}(x(k T))$ given by

$$
\mathrm{Ł}_{0}(x(k T))=c_{1} \mathrm{\Xi}_{0}\left(x_{01}\right)+c_{2} \mathrm{E}_{0}\left(x_{02}\right)+\ldots+c_{n} \mathrm{E}_{0}\left(x_{0 n}\right),
$$

in which $L_{0}\left(x_{0 i}\right)(t), t \in[k T, k T+T]$, is the zero-input response subject to the initial condition $x(k T)=x_{0 i}$, and constants $c_{i}^{\prime} s$ are calculated from the equation $x(k T)=c_{1} x_{01}+c_{2} x_{02}+\ldots$ $+c_{n} x_{0 n}$. Similar to the proof of Theorem 2, it can be shown that the tracking performance of the infinite-time tracking control is controlled by the number $N$ in such a way that $J_{k}$ approaches zero as $N$ in Eq. (14) approaches infinity for all $k \geq 0$.

An important point to be noticed about the infinite-time tracking control is that $y_{m k}$ in Eq. (16) contains the sampled system state $x(k T)$. Thus, the control input $u(t)$ in Eq. (14) also contains $x(k T)$. In fact,

$$
u(t)=v_{k}(t)-K_{k}(t) x(k T), \quad k T \leq t<k T+T,
$$

where $v_{k}(t)=U(t)\left\langle Y_{k}, Y_{k}\right\rangle^{-1}\left\langle y_{m}, Y_{k}\right\rangle^{T}$ and $K_{k}(t)=U(t)\left\langle Y_{k}\right.$, $\left.Y_{k}\right\rangle^{-1}\left\langle C(t) \Phi(t, k T), Y_{k}\right\rangle^{T}$. Equation (17) shows that the proposed tracking control actually contains a discretized linear time-varying state feedback loop. There is then the possibility that the closed-loop system becomes unstable even though the open-loop system (1) is assumed to be stable.

Before stating a sufficient condition on the closed-loop stability under the proposed control, a definition of uniform linear independence of the output data $\left\{y_{i}^{k}(t), i=1,2, \ldots, N\right\}$ is required.

Definition: The time functions $\left\{y_{i}^{k}(t), i=1,2, \ldots, N\right\}$ are uniformly linearly independent if given any $\epsilon>0$, there exists a finite time constant $T=T(\epsilon)$ such that

$$
\int_{k T}^{k T+T} Y_{k}^{T}(t) Y_{k}(t) d t>\epsilon I_{N \times N} .
$$

where $Y_{k}(t)=\left[y_{1}^{k}(t), y_{2}^{k}(t), \ldots, y_{N}^{k}(t)\right]$.

Remark: It follows immediately from the above definition that the matrix

$$
P_{k}=\int_{k T}^{k T+T} \omega(t) Y_{k}^{T}(t) Y_{k}(t) d t,
$$

which appears in Eq. (15), becomes more and more positive definite as $T$ approaches infinity in the sense that its minimum singular value approaches infinity as $T$ approaches infinity. Simulation experiences suggest that if the input data $\left\{u_{i}(t), i=1\right.$, $2, \ldots, N\}$ are chosen to be uniformly linearly independent, so are the output data. For example, $u_{i}(t)$ can be periodical time functions with different frequencies as in the simulation example in Section 6.

One can now state a stability theorem for the proposed infinite-time tracking control.

Theorem 3: Consider the system (1) and the infinite-time tracking control in Eqs. (14) and (15). If the output data $\left\{y_{i}(t)\right.$, $i=1,2, \ldots, N\}$ are uniformly linearly independent, there exists a minimum preview time $T_{\min }$, such that for all $T \geq T_{\min }$ in Eq. (14), the system state remains uniformly bounded.
Proof: See Appendix D.

The stability result in Theorem 3 is obtained regardless of the stability property of the system's zero-dynamics. Hence, the proposed infinite-time tracking control can be applied to systems with unstable zero dynamics. One can further check from the proof of Theorem 3 in Appendix D that when there are parametric uncertainties in the system, the stability claim in Theorem 3 remains true. The reason is as follows. Note that $\left\|P_{k}^{-1}(T)\right\|$ in Eq. (D4) decreases as $T$ increases because of the uniform linear independence of the output data. Since the effect of parametric uncertainties is only to make a bounded perturbation on $Q_{1}(k, T)$ and $Q_{2}(k, T)$ in Eq. (D2), the claim in Eq. (D5) still holds with the only difference that the value of $T_{\min }$ will now change due to the parametric uncertainties.

\section{Periodically Time-Varying Systems}

It is interesting to examine the proposed control design for the special case where the system matrices $A(t), B(t)$, and $C(t)$ are periodical functions of time with some period $T_{p}$. In this case, the time interval $T$ for the infinite-time tracking control (14) is chosen to be an integral multiple of $T_{p}$; that is, $T=m T_{p}$ for some positive interger $m$. It is claimed that under these conditions, the proposed control design can be completely based on a set of experimental data instead of on the exact information of system matrices. In particular, the following data will be required: the zero-input responses $L_{0}^{0}\left(x_{0 i}\right)(t)^{\prime} s, i=0,1 \ldots$, $n$, and the zero-initial-state responses $y_{i}^{0}(t)^{\prime} s=L_{u}^{0}\left(u_{i}\right)(t)^{\prime} s$, $i=0,1 \ldots, N$, on the first time interval $[0, T]$. These firsttime-interval data $L_{0}^{0}\left(x_{0 i}\right)(t)^{\prime} s$ and $L_{u}^{0}\left(u_{i}\right)(t)$ can be used for the calculation of the optimal parameter $\Theta^{*}(k)$ for all the other time intervals $k \geq 0$. In fact, based on the periodicity of the system, it will be shown that

$$
\begin{aligned}
L_{0}^{k}\left(x_{0 i}\right)(t)=L_{0}^{0}\left(x_{0 i}\right)(t), \quad L_{u}^{k}\left(u_{i}\right)(t)= & L_{u}^{0}\left(u_{i}\right)(t), \\
& \text { for all } k>0
\end{aligned}
$$

where the superscript $k$ denotes the response corresponding to the $k^{\prime}$ th time interval $[k T, k T+T]$.

Equation (18) will first be verified for the case $k=1$. The output response of the system (1) during the first time interval, subject to the initial condition $x(0)=x_{0 i}$ and the control input $u_{i}(t), t \in[0, T]$, is given by

$$
\begin{aligned}
y(t) & =C(t) \phi(t, 0) x_{0 i}+C(t) \int_{0}^{t} \phi(t, \tau) B(\tau) u_{i}(\tau) d \tau \\
& \triangleq \mathrm{Ł}_{0}^{0}\left(x_{0 i}\right)+\mathfrak{Ł}_{u}^{0}\left(u_{i}\right), \quad t \in[0, T] .
\end{aligned}
$$

The output response during the second time interval, subject to the same initial condition $x(T)=x_{0 i}$ and the control input $u_{i}(t$ $-T), t \in[T, 2 T]$, is given by

$$
\begin{aligned}
y(t+T)= & C(t+T) \phi(t+T, T) x_{0 i} \\
& +C(t+T) \int_{t}^{t+T} \phi(t+T, \tau) B(\tau) u_{i}(\tau-T) d \tau
\end{aligned}
$$$$
t \in[0, T] \text {. }
$$

From periodicity of the system, $\phi\left(t+T_{p}, \tau+T_{p}\right)=\phi(t, \tau)$, and hence

$$
\phi(t+T, \tau+T)=\phi(t, \tau),
$$

since $T=m T_{p}$. From Eq. (21) and the periodical property of the system matrices, Eq. (20) becomes

$$
\begin{aligned}
y(t+T)= & C(t) \phi(t, 0) x_{0 i} \\
& +C(t) \int_{0}^{t} \phi(t+T, v+T) B(v+T) u_{i}(v) d v \\
= & C(t) \phi(t, 0) x_{0 i}+C(t) \int_{0}^{t} \phi(t, v) B(v) u_{i}(v) d v \\
\triangleq & £_{0}^{1}\left(x_{0 i}\right)+\succeq_{u}^{1}\left(u_{i}\right), \quad t \in[0, T] .
\end{aligned}
$$




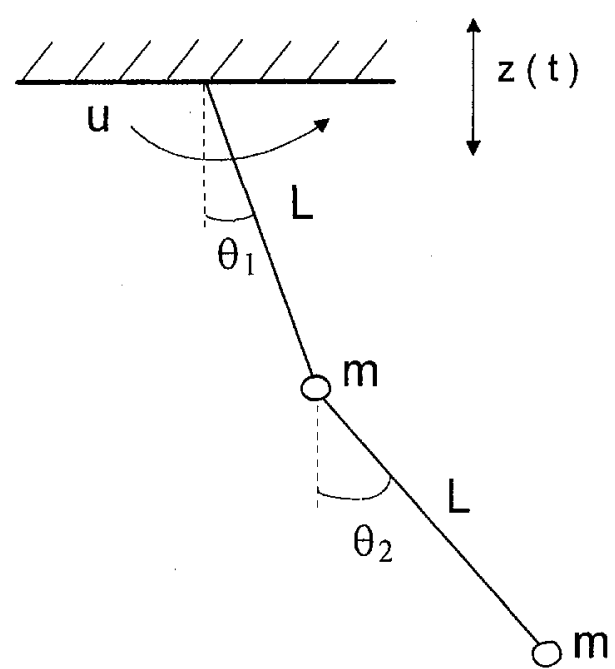

Fig. 1 Double pendulum on a moving support

Comparison of Eq. (22) with Eq. (19) shows that $L_{0}^{1}\left(x_{0 i}\right)(t)$ $=L_{0}^{0}\left(x_{0 i}\right)(t)$ and $\mathbf{七}_{u}^{1}\left(u_{i}\right)(t)=\mathbf{E}_{u}^{0}\left(u_{i}\right)(t)$. Equation (18) is thus verified for the case $k=1$. Following the same procedure, it can be shown that Eq. (18) holds for all $k$. Finally, Theorem 3 suggests that for a periodically time-varying system, there exists an integer $m^{*}$ such that for all $m>m^{*}$, the closed-loop system under the infinity-time tracking control (14) with $T=$ $m T_{p}$ remains stable.

\section{Simulation Example}

Consider a double pendulum mounted on a moving support in Fig. 1. The linearized state equation of the system is given by

$$
\dot{x}=\left[\begin{array}{cccc}
0 & 1 & 0 & 0 \\
\frac{-2}{L}(g-\ddot{z}(t)) & \frac{-3 c}{m L^{2}} & \frac{1}{L}(g-\ddot{z}(t)) & \frac{2 c}{m L^{2}} \\
0 & 0 & 0 & 0 \\
\frac{2}{L}(g-\ddot{z}(t)) & \frac{4 c}{m L^{2}} & \frac{-2}{L}(g-\ddot{z}(t)) & \frac{-3 c}{m L^{2}}
\end{array}\right] x
$$

where $x_{1}=\theta_{1}, x_{2}=\dot{\theta}_{1}, x_{3}=\theta_{2}, x_{4}=\dot{\theta}_{2}$, and the system parameters are as follows: the length of the pendulum $L=1$, weight mass $m=1$, damping coefficient $c=4$, and acceleration of gravity $g=9.81$. The support is moving up and down with an acceleration $\ddot{z}(t)=\sin (t)$. The objective is to do tracking control on the angle of the second pendulum; that is, the system output $y=x_{3}$. Notice that the system (23) with the output $y=$ $x_{3}$ has an unstable zero dynamics, and this can be verified by observing the growing trend of the system state when applying the feedback linearization control in Isidori, 1989. The reference trajectory is given by $y_{m}(t)=0.1 \sin ^{3}(t)$. The input data functions are chosen to be $u_{i}(t)=\cos ((i-1) \omega t), \omega=\pi /$ $10, i=1,2, \ldots, N$. Figure 2 , in which the dash line indicates the reference trajectory and the solid line the system output, shows the finite-time tracking performance $(T=10)$ when only 5 input data functions $(N=5)$ are used in the control. If $N$ is increased from 5 to 20 , it is seen from Fig. 3 that the tracking performance is substantially improved, and this agrees with

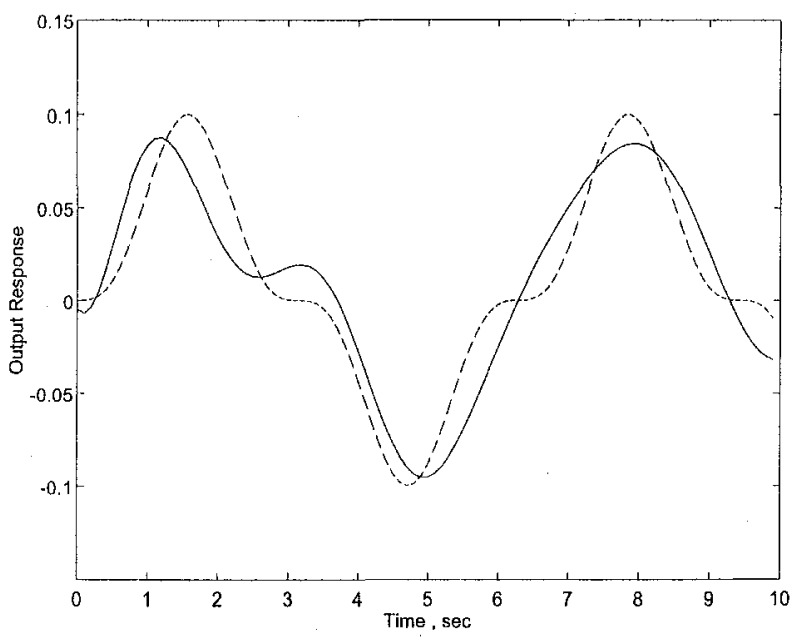

Fig. 2 Tracking performance with 5 input data functions $(N=5)$

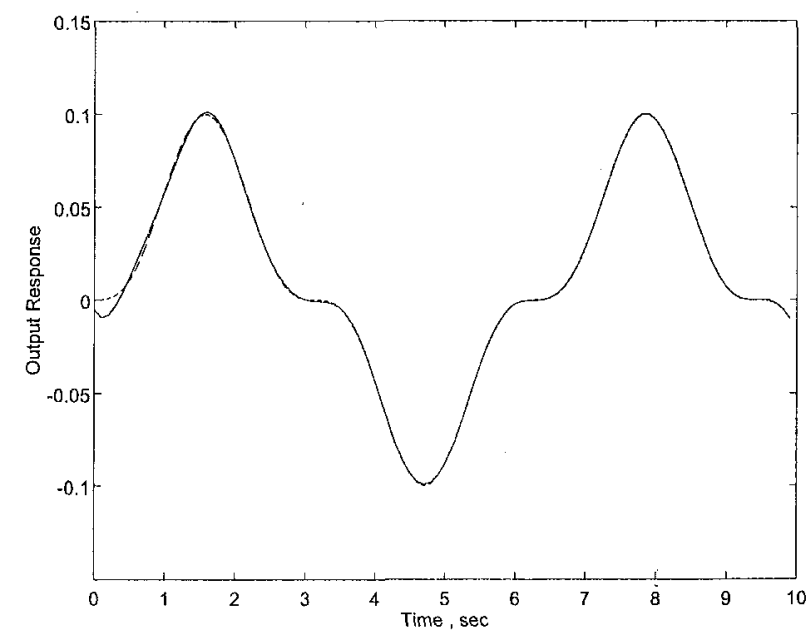

Fig. 3 Tracking performance with 20 input data functions $(N=20)$

Theorem 2 that the magnitude of the tracking error can be controlled by the number $N$. Figure 4 shows the infinite-time tracking result for the same reference trajectory, where $T=10$ and $N=20$ are used in the control (14). Figure 5 shows the system state, which confirms Theorem 3 that the system state

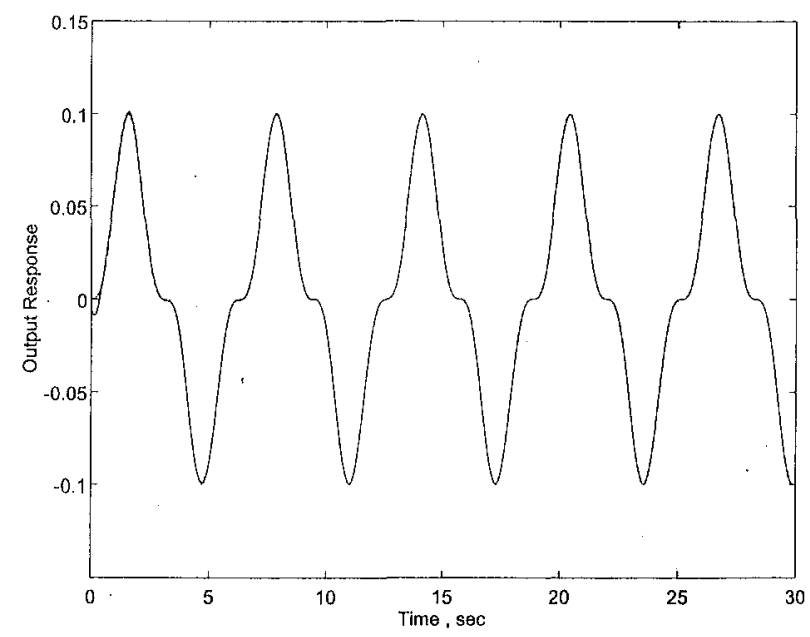

Fig. 4 Tracking performance of the infinite-time tracking control 


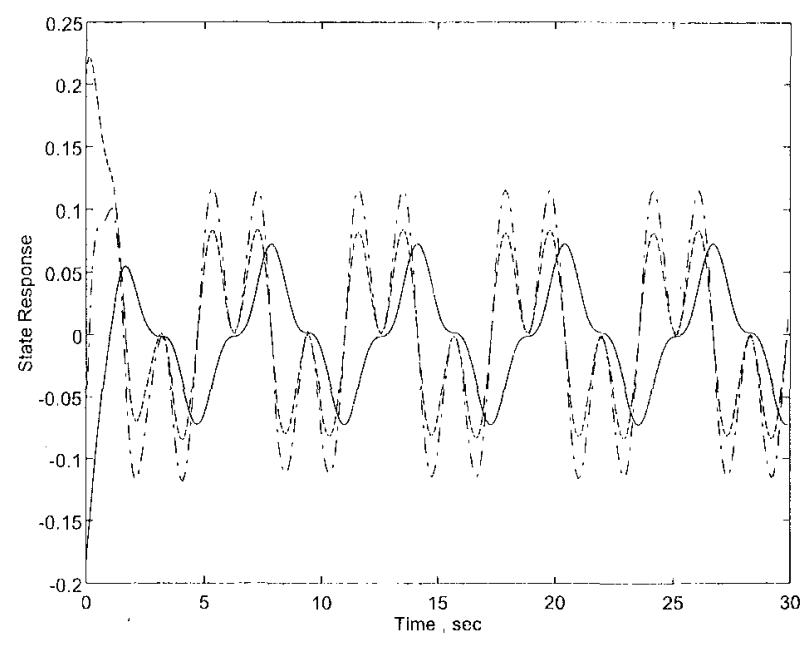

Fig. 5 State trajectories under the infinite-time tracking control

remains uniformly bounded under the proposed control. However, the control input $u(t)$ has small discontinuities at $t=k T$, $k=1,2, \ldots$, a problem that remains to be solved in the future.

\section{Conclusions}

A tracking control design for a class of LTV systems is introduced based on the assumption that a finite-time preview of the system matrices and the reference trajectory is available. The proposed design is based on a parameter optimization approach, which minimizes the $L_{2}$-norm of the output tracking error. It is shown that as long as the preview time is longer than a critical value, the closed-loop system remains stable.

\section{Acknowledgment}

This research was supported by the National Science Council in Republic of China under Contract No. NSC 81-0401-E-002554.

\section{References}

Arvanitis, K. G., and Paraskevopoulos, P. N., 1992, "Uniform Exact Model Matching for a Class of Linear Time-Varying Analytic Systems," Systems and Control Letters, Vol. 19, pp. 313-323.

Benedetto, M. D., and Lucibello, P., 1993, "Inversion of Nonlinear TimeVarying Systems," IEEE Trans. Automat. Contr., Vol. 38, pp. 1259-1264.

Choura, S., 1992, "On the Design of Finite Time Settling Control for Linear Systems," ASME JoURnAl, of DYNAMIC SYSTEMS, MEASUREMENT, AND ConTrol, Vol. 114, pp. $359-368$.

Devasia, S., and Paden, B., 1994, "Exact Output Tracking for Nonlinear TimeVarying Systems," Proceedings of Conference on Decision and Control, pp. $2346-2354$.

Gross, E., Tomizuka, M., and Messner, W., 1994, "Cancellation of Discrete Time Unstable Zeros by Feedforward Control," ASME Journal of Dynamic Systems, Measurement, and Control, Vol. 116, pp. 33-38.

Haack, B., and Tomizuka, M., 1991, "The Effect of Adding Zeros to Feedforward Controllers," ASME Journal of Dynamic Systems, MEasurement, And CONTROL, Vol. 113, pp. 6-10.

Isidori, A., 1989, Nonlinear Control Systems, Springer-Verlag, Berlin.

Kamen, E. W., 1989, "Tracking in Linear Time-Varying Systems," Proceedings of Conference on Decision and Control, pp. 263-268.

Kleinman, D., and Athans, M., 1968, "The Design of Suboptimal Linear TimeVarying Systems," IEEE Trans. Automat. Contr., Vol. 13, pp. 150-159.

Menq, C. H., and Xia, Z., 1990, "Characterization and Compensation of Discrete Time Nonminimum Phase Zeros for Precision Tracking Control," ASMF Journal of Dynamic Systems, Measurement, and CONTrol, pp. 15-23.

Rudin, W., 1974, Real and Complex Analysis, McGraw-Hill, New York, NY.

Tomizuka, M., 1987, '"Zero Phase Error Tracking Algorithm for Digital Control," ASMe Journal of Dynamic Systems, Measurement, and Control, Vol. 109 , pp. $65-68$

Tsakalis, K. S., and Ioannou, P. A., 1993, Linear Time-Varying Plants: Control and Adaptation, Prentice-Hall, Englewood Cliffs, NJ.

Zhao, H., and Chen, D., 1994, "Minimum-Energy Approach to Stable Inversion of Nonminimum Phase Systems," Proceedings of the American Control Conference, pp. 2705-2709.

\section{A P P E N D I X A}

Equation (10) can be easily proved by differentiation of the cost function (9) with respect to $\Theta$. One needs only to show that $P_{0}(\geq 0)$ is invertible due to the linear independence of the output data responses $y_{1}^{0}(t), y_{2}^{0}(t), \ldots, y_{N}^{0}(t)$. From the definition of $P_{0}=\left\langle Y_{0}, Y_{0}\right\rangle$, where $Y_{0}(t)=\left[y_{1}^{0}(t), y_{2}^{0}(t), \ldots\right.$, $\left.y_{N}^{0}(t)\right]$, given any constant vector $x \in R^{N}$, one has $x^{T} P_{0} x=$ $\int_{0}^{T} \omega(\tau)\left\|Y_{0}(\tau) x\right\|^{2} d \tau \geq 0$, and hence $x^{T} P_{0} x=0$ if and only if $Y_{0}(\tau) x=0$ for all $\tau \in[0, T]$. Since $\left\{y_{i}^{0}(t), i=1,2, \ldots\right.$, $N\}$ are linearly independent on $[0, T], Y_{0} x=0$ only if $x=0$. Therefore, it is concluded that $x^{T} P_{0} x=0$ if and only if $x=0$, and hence $P_{0}$ must be strictly positive definite.

Finally, direct substitution of $\Theta^{*}$ into $e(t)$ and $J(\Theta)$ shows that the minimal tracking error is as shown in Eq. (11), and the minimal cost is given by

$$
\begin{aligned}
J_{N}^{*} & =Y_{m 0}-V_{0} P_{0}^{-1} V_{0}^{T} \\
& =\left\langle y_{m 0}, y_{m 0}\right\rangle-\left\langle y_{m 0}, Y_{0}\right\rangle\left\langle Y_{0}, Y_{0}\right\rangle^{-1}\left\langle y_{m 0}, Y_{0}\right\rangle^{T}
\end{aligned}
$$

\section{A P P E N D I X B}

When there are $N+1$ data pairs $\left[u_{i}(t), y_{i}(t)\right]$ available, the minimal cost function achieved is given by, according to Eq. (A1),

$$
J_{N+1}^{*}=Y_{m 0}-\bar{V}_{0} \bar{P}^{-1} \bar{V}_{0}^{T},
$$

where

$$
\bar{V}_{0}=\left[\begin{array}{ll}
V_{0} & v
\end{array}\right] \in R^{1 \times(N+1)}, \quad \bar{P}=\left[\begin{array}{cc}
P_{0} & s \\
s^{T} & w
\end{array}\right] \in R^{(N+1) \times(N+1)},
$$

in which $V_{0}$ and $P_{0}$ are as in Eq. (A1), and

$$
\begin{gathered}
v=\left\langle y_{N+1}, y_{m 0}\right\rangle \in R^{1}, \quad w=\left\langle y_{N+1}, y_{N+1}\right\rangle \in R^{1}, \\
s=\left\langle Y_{0}, y_{N+1}\right\rangle \in R^{N}
\end{gathered}
$$

with $Y_{0}=\left[y_{1}^{0}, y_{2}^{0}, \ldots, y_{N}^{0}\right]$. Using the formula for block matrix inverse, the second term in $J_{N+1}^{*}$ can be expressed as

$$
\begin{aligned}
\bar{V}_{0} \bar{P}^{-1} \bar{V}_{0}^{T}= & {\left[\begin{array}{ll}
V_{0} & v
\end{array}\right] } \\
& \times\left[\begin{array}{cc}
P_{0}^{-1}+P_{0}^{-1} s \Delta^{-1} s^{T} P_{0}^{-1} & -P_{0}^{-1} s \Delta^{-1} \\
-\Delta^{-1} s^{T} P_{0}^{-1} & \Delta^{-1}
\end{array}\right]\left[\begin{array}{c}
V_{0}^{T} \\
v
\end{array}\right], \\
= & V_{0} P_{0}^{-1} V_{0}^{T}+\left(s^{T} P_{0}^{-1} V_{0}-v\right)^{T} \Delta^{-1}\left(s^{T} P_{0}^{-1} v_{0}-v\right)
\end{aligned}
$$

where $\Delta=\left\langle y_{N+1}, y_{N+1}\right\rangle-s^{T} P_{0}^{-1} s \in R^{+}$(it is shown in Appen$\operatorname{dix} C$ that $\Delta$ is a positive number and hence invertible). Since

$$
\begin{aligned}
s^{T} P_{0}^{-1} V_{0}-v & =s^{T} \Theta_{N}^{*}-v=\left\langle y_{N+1}, Y_{0}\right\rangle \Theta_{N}^{*}-\left\langle y_{N+1}, y_{m 0}\right\rangle \\
& =\left\langle y_{N+1}, Y_{0} \Theta_{N}^{*}-y_{m 0}\right\rangle=-\left\langle y_{N+1}, e_{N}^{*}\right\rangle,
\end{aligned}
$$

where Eq. (10) is used to obtain the first equality, and Eq. (11) the last equality, subtracting Eq. (A1) from Eq. (B1) results in

$$
J_{N+1}^{*}-J_{N}^{*}=-\left\langle y_{N+1}, e_{N}^{*}\right\rangle^{T} \Delta^{-1}\left\langle y_{N+1}, e_{N}^{*}\right\rangle .
$$

It then follows from the Hypothesis in (12) and the positiveness of $\Delta$ that $J_{N+1}^{*}-J_{N}^{*}<0$.

To prove Eq. (13), note that if the sequence $y_{i}(t)$ is complete, the set of all finite linear combinations of $y_{i}(t)$ is dense in the $L^{2}$ space on $[0, T]$ (Theorem 4.18, Rudin, 1974). By quoting the Approximation Problem in Section 4.15 and Theorem 4.16 in Rudin (1974) it is concluded that given any desired $\epsilon>0$, there exists a number $N$ such that $J_{N}^{*}<\epsilon$. This concludes the proof for Eq. (13). 


\section{A P P E N D I X C}

It will be shown that the number

$$
\Delta=\left\langle y_{N+1}, y_{N+1}\right\rangle-\left\langle y_{N+1}, Y\right\rangle\langle Y, Y\rangle^{-1}\left\langle y_{N+1}, Y\right\rangle^{T}
$$

in Eq. (B2) is a positive number if $\left\{y_{1}, y_{2}, \ldots, y_{N+1}\right\}$ are linear independent time functions. First, to show that $\Delta$ is nonnegative, notice that $\Delta$ in Eq. (C1) has exactly the same form as $J^{*}$ in Eq. (A2) with $y_{N+1}$ in $\Delta$ acting as $y_{m 0}$ in $J^{*}$. Thus, $\Delta$ can be treated as the minimal cost function $J^{*}$ with a reference trajectory $\tilde{y}_{m}$ given by

$$
y_{m 0}=\tilde{y}_{m}-七_{0}\left(x_{0}\right)=y_{N+1} \text {, or } \tilde{y}_{m}=y_{N+1}+\bigsqcup_{0}\left(x_{0}\right) .
$$

Hence, $\Delta$ must be non-negative. Second, a contradiction argument will be used to show that $\Delta$ is nonzero. Assuming that $\Delta$ $=0$, it follows from the previous statement that

$$
\begin{aligned}
\Delta=J^{*}=0 & =\int_{0}^{T} e^{*^{2}}(t) d t \\
& =\int_{0}^{T}\left[\tilde{y}_{m}(t)-y^{*}(t)\right]^{2} d t \\
& =\int_{0}^{T}\left[y_{N+1}(t)+\mathrm{七}_{0}\left(x_{0}\right)-y^{*}(t)\right]^{2} d t .
\end{aligned}
$$

Consequently,

$$
\begin{aligned}
y_{N+1}(t) & =-\mathrm{Ł}_{0}\left(x_{0}\right)+y^{*}(t)=-\mathrm{七}_{0}\left(x_{0}\right)+\mathrm{七}_{0}\left(x_{0}\right)+\mathrm{七}\left(u^{*}\right) \\
& =\mathrm{L}\left(\sum \theta_{i}^{*} u_{i}\right)=\sum_{i=1}^{N} \theta_{i}^{*} y_{i}(t) \quad \forall t \in[0, T] .
\end{aligned}
$$

However, the last equation is a contradiction with the fact that $\left\{y_{1}, y_{2}, \ldots, y_{N+1}\right\}$ are linearly independent. It is thus concluded that $\Delta \neq 0$.

\section{A P P E N D I X D}

Discretize the closed-loop system (1) and (14) with a sampling period $T$ to obtain

$$
x[(k+1) T]=\bar{A}(k) x(k T)+\bar{D}(k), \quad k=0,1,2, \ldots
$$

where

$$
\begin{array}{r}
\bar{D}(k)=\int_{k T}^{(k+1) T} \Phi[(k+1) T, \tau] B(\tau) U(\tau) P_{k}^{-1} \\
\times \int_{k T}^{(k+1) T} \omega(s) Y_{k}^{T}(s) y_{m}(s) d s d \tau, \\
\bar{A}(k)=Q_{3}(k, T)-Q_{1}(k, T) P_{k}^{-1}(T) Q_{2}(k, T), \quad(\mathrm{D} 2) \\
Q_{1}(k, T)=\int_{k T}^{(k+1) T} \Phi[(k+1) T, \tau] B(\tau) U(\tau) d \tau \in R^{n \times N} \\
Q_{2}(k, T)=\int_{k T}^{(k+1) T} \omega(s) Y_{k}^{T}(s) C(s) \Phi(s, k T) d s \in R^{N \times n} \\
Q_{3}(k, T)=\Phi[(k+1) T, k T] .
\end{array}
$$

Since the open-loop system (1) is exponentially stable, and the input data $U(t)$ and the output data $Y_{k}(t)$ are all uniformly bounded, one can show that for all integer $k$ and any time interval length $T$,

$$
\begin{gathered}
\left\|Q_{1}(k, T)\right\| \leq M_{1}, \quad\left\|Q_{2}(k, T)\right\| \leq M_{2}, \\
\left\|Q_{3}(k, T)\right\| \leq m e^{-\sigma T},
\end{gathered}
$$

for some positive constants $M_{1}$ and $M_{2}$, and $m$ and $\sigma$ are as in Eq. (2). Taking the matrix norm of $\bar{A}(k)$ in Eq. (D2) and using Eq. (D3) result in

$$
\|\bar{A}(k)\| \leq m e^{-\sigma T}+M_{1} M_{2}\left\|P_{k}^{-1}(T)\right\| .
$$

The Remark before Theorem 3 shows that $\left\|P^{-1}\right\|$ is a strictly decreasing function of $T$. Hence, according to Eq. (D4), there exists a $T_{\min }$ such that

$$
\|\bar{A}(k)\|<1, \quad \forall k>0 \quad \text { and } \quad \forall T \geq T_{\min } .
$$

Finally, uniform boundedness of $x(k T)$ can be concluded by invoking the contraction mapping Theorem to Eq. (D1), and by noting that $\bar{D}$ in Eq. (D1) is uniformly bounded since it is assumed that the input data $U(t)$ and hence the output data $Y_{k}(t)$ are uniformly bounded. 